\title{
Visualization Methods for Personal Photo Collections: Browsing and Searching in the PhotoFinder
}

\author{
Hyunmo Kang, Ben Shneiderman \\ Department of Computer Science, Human-Computer Interaction Laboratory \\ University of Maryland at College Park \\ College Park, MD 20742 USA \\ $+13014052725$ \\ $\{$ kang, ben\}@cs.umd.edu
}

\begin{abstract}
Software tools for personal photo collection management are proliferating, but they usually have limited searching and browsing functions. We implemented the PhotoFinder prototype to enable non-technical users of personal photo collections to search and browse easily. PhotoFinder provides a set of visual Boolean query interfaces, coupled with dynamic query and query preview features. It gives users powerful search capabilities. Using a scatter plot thumbnail display and drag-and-drop interface, PhotoFinder is designed to be easy to use for searching and browsing photos.
\end{abstract}

Keywords : PhotoFinder, user interface, dynamic query, query preview, search, browsing, Boolean query, digital photo library.

\section{INTRODUCTION}

Digital cameras, scanners and personal computers are now common. But as collections grow in size, the need to organize, search, and browse digital photos increases [1]. There are many personal photo collection management tools available either commercially or non-commercially. A search with terms "digital photo" and "software download" on ZDNet.com or CNet.com will return many hits pointing to software entries. These software products vary substantially in terms of features and quality. We find what most of these products lack are easy-to-learn and efficient searching and browsing capabilities, especially for collections of photos. This has been a long-standing problem for digital photo library tools. PhotoFinder was built as an attempt to address some of these problems. We implemented some novel widgets that can be used to do powerful Boolean queries. A variety of information visualization techniques, such as dynamic queries and query previews are employed to provide facilities to search and browse easily and effectively. We also designed interfaces to cope with missing data. This paper describes the features implemented in the PhotoFinder prototype and discusses their advantages and disadvantages.

\section{DESIGN OUTLINES}

Influenced by some earlier projects at the Human Computer Interaction Lab.(HCIL), University of Maryland at College Park, our design for PhotoFinder employs the following strategies:

- powerful Boolean queries to select desired photos

- dynamic queries to rapidly filter and view results

- query previews to show cardinality of result sets

- special fields to handle missing data for an attribute

\subsection{User Interface Strategies}

We wanted to use a direct manipulation interface with visual representation for the search space. This allows rapid, incremental, and reversible exploratory actions. We wanted to construct visual widgets to simplify the operations for searching and browsing: e.g. pointing and selecting will be favored over typing. The interface should produce immediate, continuous and visual feedback of search/query results.

The PhotoFinder prototype has a three-window strategy: a search tool window (top level), a thumbnail browser window (intermediate level), a detail viewer window (bottom level). This follows the tried and tested visual information seeking strategy: "Overview first, zoom and filter, and then details on demand"[2]. We combined dynamic query techniques with query previews that show the cardinality of result sets, thereby guiding users to avoid zero-hit queries.

The three-window strategy tightly coupled displays to form a coherent view to the data that users are searching and browsing.

\subsection{Exploring PhotoFinder}

PhotoFinder allows users to explore a library that consists of multiple collections. Users begin with the collection browser to select a single collection for detailed study. The collection browser shows a representative photo for each collection (Figure 1). There are three major parts of the PhotoFinder:

The search tool window contains a series of tabbed panels of dialog boxes for setting query attributes. On each tabbed panel, an attribute distribution chart shows the number of hits for those attributes. Innovative multiple two-sided slider bars are used for query range selections. The overview window gives users three filtering mechanisms to display photo thumbnails: show all hits for the current query (default behavior), show hits for each independent attribute query (if "Show Thumbnail" button is clicked), and show hits for the corresponding histogram bar (if a histogram bar is clicked and highlighted).

There are two major display modes for the thumbnail browser window: sequential display and scatter plot display. Zooming is provided to better use the display space and for the ease of navigation. Users can also just point and click to locate and select an individual picture or a set of pictures in the thumbnail browser window.

The detail viewer window displays the full-size picture selected from thumbnail browser and its annotation. A slide show function is also implemented to allow users to display a set of selected pictures one after another. The detail viewer window also provides facilities for annotating a single picture. 


\subsection{Implementation Environment}

PhotoFinder is implemented using Microsoft Visual Basic 6.0. The sample photo library was implemented and maintained as a Microsoft Access database. Through ODBC, PhotoFinder is able to connect to and query the database.

\section{SYSTEM FEATURES}

\subsection{Boolean Query and Search Interface}

Enabling users to do Boolean queries visually is a major humancomputer interaction topic. Many approaches have been suggested with different degrees of implementation difficulties and different degrees of success [5][8]. To provide full Boolean query power in a visual form is difficult. In our experience, users of digital photo libraries tend to perform Boolean queries for searching pictures with some limited patterns [6]. Namely, users tend to use Boolean queries as follows:

- make OR queries within an attribute;

- make AND queries across different attributes;

For example, users may want to find pictures "of Anne OR Bob" that (AND) "were taken in 1999", but not pictures "of Anne OR those taken in Las Vegas".
Our approach for disjunctive (OR) queries within attributes is to use MTS (Multiple-Two-Sided) slider bars for each attribute. The MTS slider bar enables disjunctive range selections (e.g. 2-4, 7-9, 14-21). Traditional double-sided slider bars give the users the ability to select just one range. Using MTS, users can specify any number of disjunctive ranges of one attribute (Figure 2). Eleven tabbed panels are used to represent query for different attributes (10 attributes plus one for "All" attributes). The relationship among these attributes in forming a query is by default conjunctive (AND). The "All Attributes" tabbed panel shows all the query parameter settings in a compact display. Histograms are used to show query results for each individual attribute.

The yellow part of each histogram bar (bright part in black and white) indicates the "hits" of query results. The gray part of the histogram bar indicates the "non-selected photos". Although the relationship among query attributes defaults to be conjunctive, users can still form complicated queries involving disjunctive relations among attributes if they so desire. This is achieved by sequentially performing different queries and merging the query results.

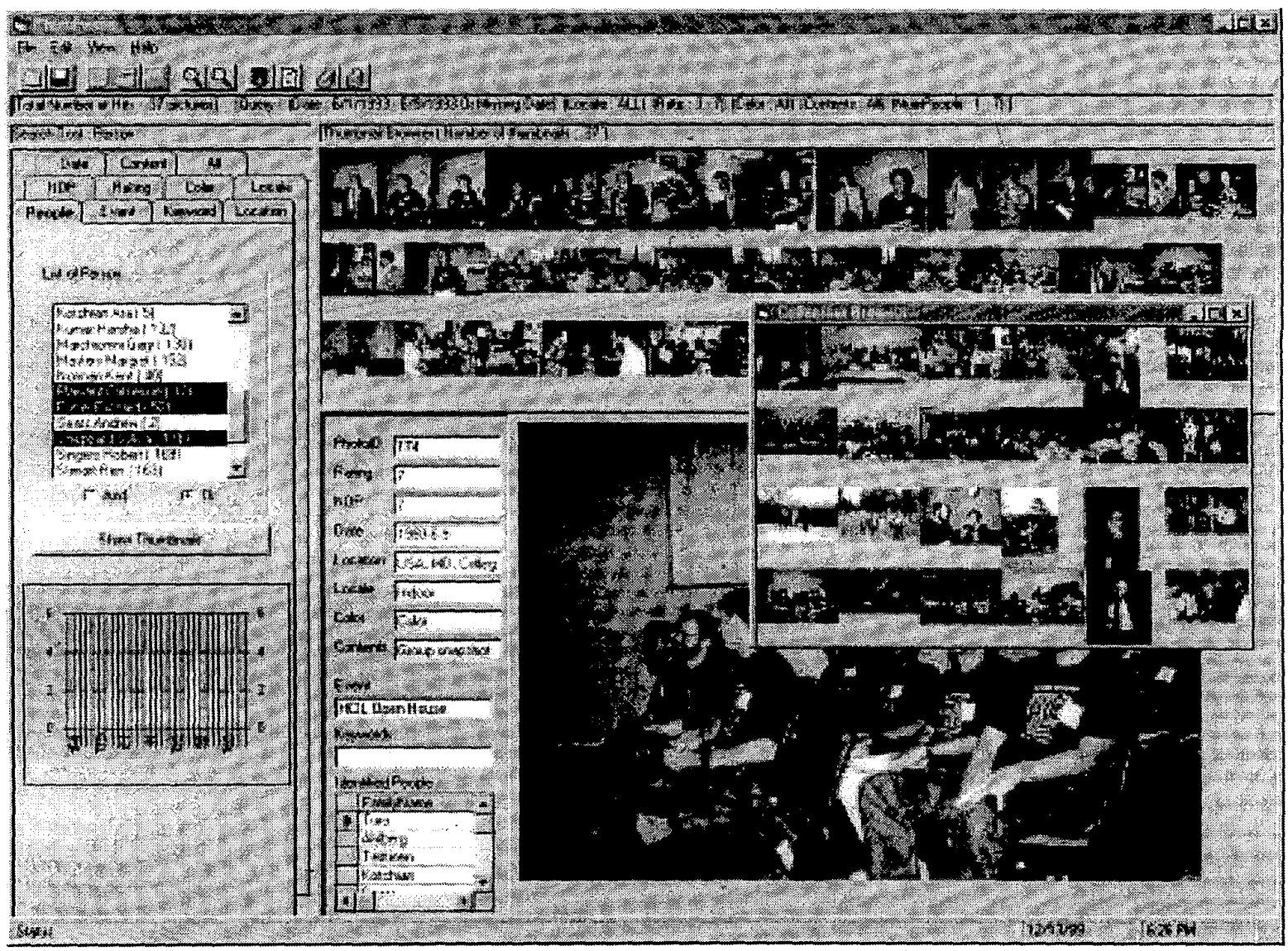

Figure 1. PhotoFinder (with search tool, thumbnail browser, and detail viewer windows) plus the overlaid Collection Browser to select a set of photos to explore. 


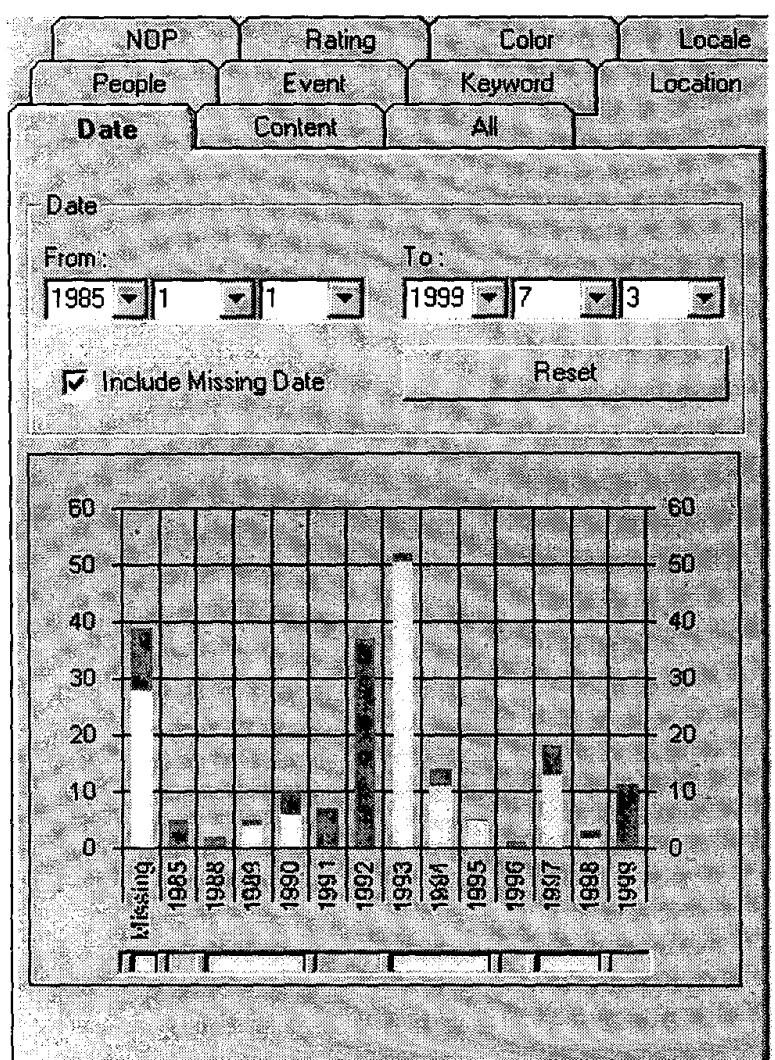

Figure 2. Examples of Dynamic Query Preview. The slider bars under the histograms are MTS slider bars where multiple disjunctive range selections can be specified by using multiple range sliders. On the left, a separate attribute value is used to represent data with missing a date.

\subsection{Dynamic Queries \& Query Previews}

Dynamic query techniques have proved to be comprehensible and effective [2][4]. Immediate feedback often provides more information than statically displayed query results. In PhotoFinder, dynamic queries are also used to enhance the searching capabilities. Query results are updated in real time for previews in the search panel, and the thumbnail browser window is also updated in real time for each query setting change. Dynamic filtering is made possible by clicking on individual histogram bars to further filter the query results. When this happens, only pictures with that particular histogram bar value will be shown. In all attribute panels, query result histograms are updated to reflect the effect of query changes.

There are also many advantages of query previews which show the distribution of data [7]. In PhotoFinder, we provided a histogram chart for each attribute as a query preview tool. The users can see the distribution of pictures for each attribute in advance and therefore gain a better understanding of the photo collection. The histogram chart also gives users immediate feedback of the number of hits for the query they are performing. Query parameters can then be adjusted to refine the query. It presumably will prevent users from performing zero-hit queries.
Missing data are displayed as an additional value in the histogram (Figure 2, left side). Therefore there is no loss of information in visualizing the contents of the collection.

\subsection{Multiple Displays in a Thumbnail Browser}

The thumbnail browser is designed to hold and display the query result for users to browse and select photos for detailed viewing. By the nature of the complexity of the photo attributes, no single way of displaying the thumbnails will fit all users' needs. Therefore our browser has multiple displays of thumbnails.

In the sequential display of photos, one picture is placed after another. Due to different sizes and orientations of photos, a large portion of the display space is wasted white space. This is the non-compact mode, which is the default mode. If "Compact" mode is selected, our heuristics will re-order the thumbnails so that pictures of the same size and orientations will be placed together to reduce the wasted white space. Figures 3a-b show the effect of non-compact and compact mode of sequential display. The compact mode is especially useful when the order of the photos is not important to users so that the display space can be better utilized.

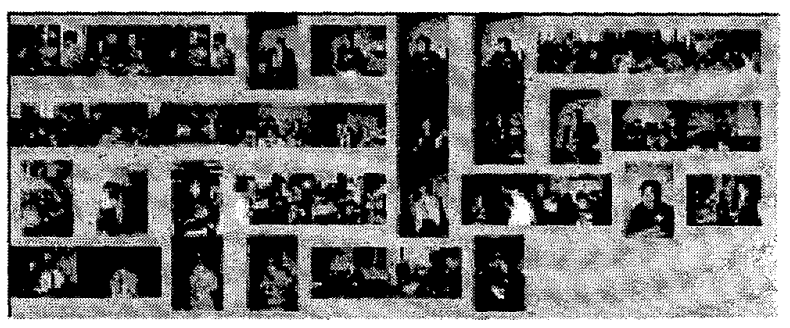

Figure 3a. Non-Compact Mode of Sequential Display

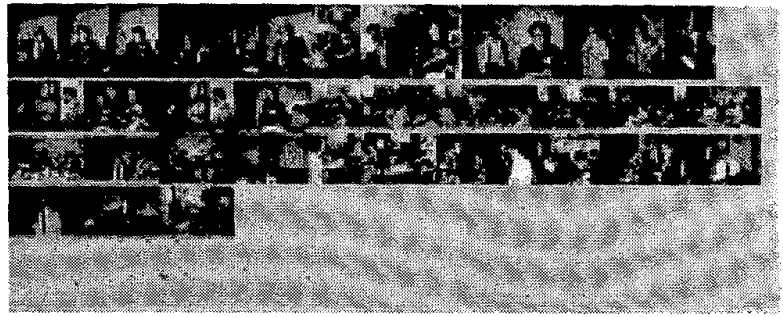

Figure 3b. Compact Mode of Sequential Display

Users can choose to have a scatter plot display for the thumbnail pictures. Like Spotfire (www.spotfire.com), such a scatter plot display, coupled with the flexibility of choosing attributes for the axes, often provides users with quick and insightful understanding of the pictures displayed (Figure 4). By choosing different axes, the distribution of pictures can reveal patterns that are difficult to find in a textual tabular display.

\subsection{Drag and Drop to the Detail Viewer Window}

From the thumbnail browser window, users can point and click to select a single picture or use a rubber rectangle to select a group of pictures. After the selection is made, users can drag and drop the selection into the detail viewer window for viewing individual photos or a slide show. Such an interface should be easy to use, and we believe it makes browsing photos more enjoyable. Figure 5 shows how drag and drop works for the detail viewer window. 


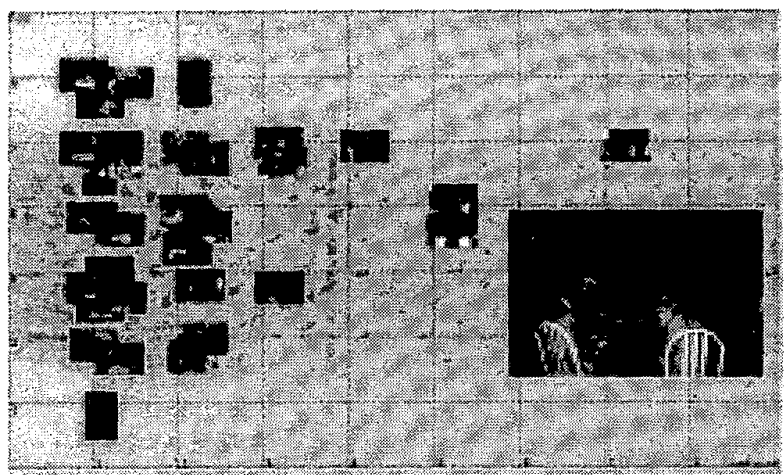

Figure 4. Scatter Plot Display of Thumbnail Browser with Zooming Function. The $x$-axis is number of people in a picture and the $y$-axis is the picture rating $(1-9,9$ is best). While most pictures in this collection have only a few people in it, the one in the northeast corner has 7 people. The distribution of pictures in this scatter plot also shows that pictures of multiple people tend to have higher ratings.

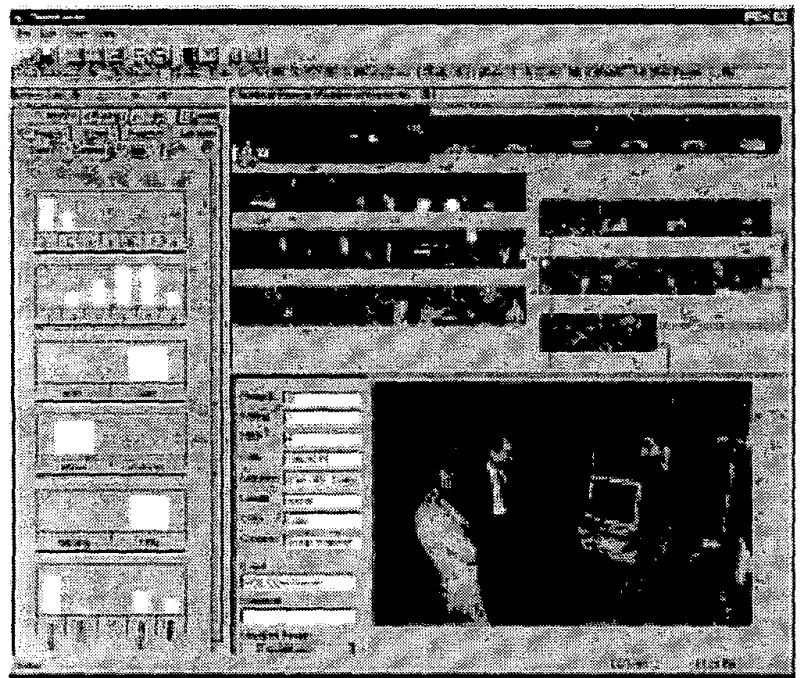

Figure 5. Drag and Drop to the Detail Viewer Window: one picture is shown in the detail viewer window, while a group selection is performed in the thumbnail browser. The selection is being dragged to the detail viewer window to create a slide show.

\section{FUTURE WORK}

We feel our design and implementation of PhotoFinder have many advantages over most of the personal photo collection management products currently available. Still, to make a really good product for home users to organize, browse and search their growing digital photo library is hard. We realize PhotoFinder has many limitations. There is still a lot of work needed in terms of features and interfaces. Currently, we have not integrated PhotoFinder with a proper Collection Browser. To combine PhotoFinder with a Collection Browser will give users a more complete solution to their personal photo library organization problem. Planned future work includes the implementation of a histogram display in the thumbnail browser. We also have plans to implement an automatic annotation mechanism so that the users can just use a mouse to click, drag and drop to make annotations instead of repetitively typing in text fields. These techniques can be further combined with functionality for annotation of single photos, groups of selected photos, or entire collections of photos. Our future work includes user studies of PhotoFinder and controlled experiments [3].

\section{CONCLUSION}

We implemented PhotoFinder to provide better searching and browsing capabilities than in existing software tools. By using a novel attribute selection query interface, PhotoFinder gives users nearly full Boolean query power with high ease of use. Information visualization techniques, such as dynamic query and query preview, further strengthen the search capabilities of PhotoFinder. We believe that multiple forms of thumbnail display and a drag-and-drop interface make PhotoFinder an enjoyable and powerful photo-browsing tool.

Acknowledgements: We appreciate the partial support of Intel, and input from colleagues: Ben Bederson, Hilary Browne, Todd Carlough, Tammara Combs, Hatim Khan, Manav Kher, Catherine Plaisant, and Jin Tong.

\section{REFERENCES}

[1] Allan Kuchinsky, Celine Pering, Michael L. Creech, Dennis Freeze, Bill Serra, Jacek Gwizdka, "FotoFile: A Consumer Multimedia Organization and Retrieval System", Proc. ACM CHI99 Conference on Human Factors in Computing Systems, pp. 496-503, May, 1999.

[2] C. North, B. Shneiderman, C. Plaisant, "User Controlled Overviews of an Image Library: A Case Study of the Visible Human", Proc. ACM Digital Libraries '96 Conference, ACM Press, New York, pp. 74-82, 1996.

[3] Joemon M.Jose, Jonathan Furner, and David J.Harper, "Spatial querying for image retrieval: a user-oriented evaluation", Pro.e 21st Annual International ACM SIGIR88 Conference on Research and Development in Information Retrieval, pp. 232-240, August, 1998.

[4] Ken Fishkin, Maureen C. Stone, "Enhanced Dynamic queries via Movable Filters", Proc. ACM CHI95 Conference on Human Factors in Computing Systems, pp. 415-420, May, 1995.

[5] Michael Chui, "Pattern, Procedurality \& Pictures: Factors Affecting Boolean Query Interface Design for the Web", Proc. ACM SIGIR99. http://www.cs.indiana.edu/ mchui/sigir $99 /$

[6] Sharon R. Garber, Mitch B. Grunes, "The Art of Search: A Study of Art Directors", Proc. ACM CHI92 Conference on Human Factors in Computing Systems, pp. 157-163, 1992.

[7] Stephan Greene, Gary Marchionini, Catherine Plaisant, and Ben Shneiderman, "Previews and Overviews in Digital Libraries: Designing Surrogates to Support Visual Information Seeking", University of Maryland Technical Report CS-TR-3838, 1997.

[8] Sylvia Willie, "Query Context: Would a graphical interface help?", School of Information Systems, Queensland University of Technology

http://www.icis.qut.edu.au/ willie/My Papers/ozchi-94.html 